PROCEEDINGS OF THE

AMERICAN MATHEMATICAL SOCIETY

Volume 127, Number 5, Pages 1437-1441

S 0002-9939(99)04997-7

Article electronically published on January 29, 1999

\title{
ON THE NORM OF THE PRODUCT OF LINEAR FUNCTIONALS
}

\author{
C. BENÍTEZ, MANUEL FERNÁNDEZ, AND MARÍA L. SORIANO
}

(Communicated by David R. Larson)

\begin{abstract}
Real inner product spaces are characterized by the points at which the homogeneous 2-polynomials that are products of equal-norm linear functionals attain their norm.
\end{abstract}

\section{INTRODUCTION}

Let $E$ be a real normed space of dimension $\geq 2$ and let $S$ be its unit sphere. For $u \in S$ let $J u$ denote the set of norm-1 linear functionals $f$ on $E$ such that $f(u)=1$. M.M. Day [3], [1, p. 85], proved that $E$ is an inner product space (i.p.s.) if and only if, for any $u, v \in S$ and $f \in J u, g \in J v$, the real function $x \in S \rightarrow f(x)+g(x) \in \mathbb{R}$ attains its maximum at the point $\|u+v\|^{-1}(u+v)$, i.e.,

$$
(f+g)(u+v)=\|f+g\|\|u+v\| .
$$

We prove that (1) can be modified to

$$
(g(u+v) f+f(u+v) g)(u+v)=\|g(u+v) f+f(u+v) g\|\|u+v\|,
$$

and we obtain from this a similar result for products, instead of sums, of linear functionals, namely, $E$ is an i.p.s. if and only if, for any $u, v \in S$ and $f \in J u$, $g \in J v$, the real function $x \in S \rightarrow f(x) g(x) \in \mathbb{R}$ attains its maximum at the point $\|u+v\|^{-1}(u+v)$.

Suppose now that (1) is modified to

$$
\forall r \in \mathbb{R}, \exists t \in \mathbb{R}:(f+r g)(u+t v)=\|f+r g\|\|u+t v\| .
$$

Then, it is easy to see that (3) is fulfilled by real i.p.s.'s and it is obvious that it is also fulfilled by every real normed space of dimension 2. However, R. C. James [4], [1, p. 104], showed that (3) characterizes real i.p.s.'s of dimension $\geq 3$.

We prove that $(3)$ can be modified to

$$
\exists r>0, \exists t>0:(f+r g)(u+t v)=\|f+r g\|\|u+t v\|,
$$

and, making use of this result, we also prove that real i.p.s.'s of dimension $\geq 3$ are characterized by the fact that $x \in S \rightarrow f(x) g(x) \in \mathbb{R}$ attains its maximum at some point $\|u+t v\|^{-1}(u+t v)$, with $t>0$.

Received by the editors August 24, 1997.

1991 Mathematics Subject Classification. Primary 46C15, 46B20.

Key words and phrases. Characterization of inner product spaces.

(C)1999 American Mathematical Society 


\section{RESUlts}

Proposition 1. A real normed space $E$ of dimension $\geq 2$ is an i.p.s. if and only if, for any $u, v \in S$ and $f \in J u, g \in J v$,

$$
2 f(u+v) g(u+v)=\|g(u+v) f+f(u+v) g\|\|u+v\| .
$$

Proof. If $E$ is an i.p.s., then $f(x)=\langle x, u\rangle, g(x)=\langle x, v\rangle$ and the rest follows easily. Furthermore, since $f(u+v)=g(u+v) \geq 0$, the above is the straightforward part of Day's result (1).

To prove the converse, it suffices to consider the 2-dimensional case, i.e., the real plane $\mathbb{R}^{2}$ endowed with a norm whose unit sphere is the convex symmetric curve $S$.

First we prove that $S$ is rotund and smooth (a curve without segments and corners).

Suppose that a segment $[v, w]$ is contained in $S$. Then there exist $g \in J v \cap J w$ and $u \in S$ such that $g(u)=0$. Condition (2) applied both times to $f \in J u$ and $g$ gives

$$
\begin{aligned}
2 f(u+v) & =\|f+f(u+v) g\|\|u+v\|, \\
2 f(u+w) & =\|f+f(u+w) g\|\|u+w\|,
\end{aligned}
$$

from which it follows that

$$
\begin{aligned}
& 2 f(u+v)\|u+w\| \geq(f(u+w)+f(u+v))\|u+v\|, \\
& 2 f(u+w)\|u+v\| \geq(f(u+v)+f(u+w))\|u+w\| ;
\end{aligned}
$$

hence, $4 f(u+v) f(u+w) \geq(f(u+w)+f(u+v))^{2}$. Therefore, $f(v)=f(w)$ and $v=w$, as we wished to show.

Suppose now that $S$ has a corner at $v$, i.e., that there exist $g, h \in J v, g \neq h$. Then, for any $u \in S$ and $f \in J u$, the two functionals $g(u+v) f+f(u+v) g$ and $h(u+v) f+f(u+v) h$ are non-proportional, but attain their norms at the same point $\|u+v\|^{-1}(u+v)$, which is in contradiction with the well-known fact that $S$ has, at most, a countable set of corners.

Finally, we shall prove that $S$ is an ellipse. To be exact, we shall prove that $S$ is symmetric (oblique symmetry, in general) with respect to any of its diameters. Since $S$ is smooth we can write $f=J u$ instead of $f \in J u$.

For a given $w \in S$ and $h=J w$, let $\bar{w} \in S$ and $\bar{h}=J \bar{w}$ be such that $\bar{h}(w)=0$ and $h(\bar{w}) \geq 0$. Since $S$ is rotund and $h(\bar{w}) \geq 0$, for any $u \in S$ between $\bar{w}$ and $w$ there is a unique $t>0$ such that $\|u-t \bar{w}\|=1$. Denote $v=v(u)=u-t \bar{w}$, applying the hypothesis to $f=J u$ and $-g=J(-v)$ we obtain that

$$
-2 f(u-v) g(u-v)=\|g(u-v) f+f(u-v) g\|\|u-v\|,
$$

or, equivalently,

$$
-2 f(\bar{w}) g(\bar{w})=\|g(\bar{w}) f+f(\bar{w}) g\| .
$$

Since $S$ is smooth, we have

$$
\bar{h}=\frac{1}{2}\left(\frac{1}{f(\bar{w})} f+\frac{1}{g(\bar{w})} g\right) ;
$$

hence,

$$
f(w) g(\bar{w})+f(\bar{w}) g(w)=0 .
$$

To simplify our final arguments, we can assume that $\bar{w}=(1,0)$ and $w=(0,1)$. (Remember that we wish to show that $S$ is an ellipse character that remains unchanged under isomorphisms of the plane.) Then, since $S$ is rotund and smooth, 
the set of points of $S$ that are between $\bar{w}$ and $w$ is the graph of a continuous function $\varphi:[0,1] \rightarrow \mathbb{R}$, differentiable on $(0,1)$, and the set of the points of $S$ that are between $-\bar{w}$ and $w$ is the graph of an analogous function $\psi:[-1,0] \rightarrow \mathbb{R}$.

Then, if $u=(x, \varphi(x))$, the corresponding point $v=v(u)=(\xi(x), \psi(\xi(x)))$ is such that $\psi(\xi(x))=\varphi(x)$. Since $\varphi^{\prime}(x)=-f(\bar{w})(f(w))^{-1}$ and $\psi^{\prime}(\xi(x))=$ $-g(\bar{w})(g(w))^{-1},(5)$ implies that $\varphi^{\prime}(x)=-\psi^{\prime}(\xi(x))$. Therefore, $\xi$ is differentiable and we have that

$$
\psi^{\prime}(\xi(x)) \xi^{\prime}(x)=\varphi^{\prime}(x)=-\psi^{\prime}(\xi(x)),
$$

from which it follows that $\xi(x)=-x+c$. Since $\psi(-1)=\varphi(1)$, we obtain that $c=0$; hence, $S$ is symmetric with respect to the arbitrary initial diameter $\{t w:|t| \leq 1\}$, as we wished to show.

The arguments of the above proof also give a direct proof of Day's characterization (1), which is simpler than Day's original proof and not based on the deep result of M. Baronti [2], which is used in the proof that appears in [1, p. 85].

Proposition 2. A real normed space $E$ is an i.p.s. if and only if, for any $u, v \in S$ and $f \in J u, g \in J v$, the real function $x \in S \rightarrow f(x) g(x) \in \mathbb{R}$ attains its maximum at the point $\|u+v\|^{-1}(u+v)$.

Taking into account that $-g \in J(-v)$, this implies that either

$$
(f g)(u+v)=\|f g\|\|u+v\|^{2}, \quad \text { or }-(f g)(u-v)=\|f g\|\|u-v\|^{2} .
$$

Proof. Suppose that $E$ is an i.p.s.. Then $f(x)=\langle x, u\rangle, g(x)=\langle x, v\rangle$, and everything reduces to the simple fact that the function

$$
t \in \mathbb{R} \rightarrow \frac{\langle u+t v, u\rangle\langle u+t v, v\rangle}{\langle u+t v, u+t v\rangle}
$$

attains its maximum at $t=1$.

Conversely, let $w \in S$ be such that $f(w)>0$ and $g(w)>0$ (the case $f(w)<0$ and $g(w)<0$ is analogous).

On the one hand, it is easy to see that the set

$$
C_{w}=\{x \in E: f(x)>0, g(x)>0, f(x) g(x) \geq f(w) g(w)\}
$$

is convex.

On the other hand, we shall prove that

$$
H_{w}=\{x \in E: g(w) f(x-w)+f(w) g(x-w)=0\}
$$

is the only closed hyperplane that supports $C_{w}$ at $w$.

First, suppose that $H_{w}$ does not support $C_{w}$. Then, there is $x \in E$ such that

$$
f(x) g(x)>f(w) g(w)>0, \quad g(w) f(x-w)+f(w) g(x-w)=0,
$$

which implies

$$
g(w) f(x)+f(w) g(x)=2 f(w) g(w)<f(w) g(w)+f(x) g(x),
$$

i.e., $f(x-w) g(x-w)>0$, which is not possible.

Suppose now that the continuous linear functional $h$ is not a multiple of $g(w) f+$ $f(w) g$. Then there is $y \in S$ such that

$$
h(y)=0<g(w) f(y)+f(w) g(y)
$$


hence, $h(w+t y)=h(w)$ for any $t \in \mathbb{R}$, but, for $t>0$ sufficiently small,

$$
f(w+t y) g(w+t y)>f(w) g(w) .
$$

In other words, the closed hyperplane $h(x)=h(w)$ does not support $C_{w}$.

Therefore, if the function $x \in S \rightarrow f(x) g(x) \in \mathbb{R}$ attains its maximum at $w \in S$, then the only closed hyperplane that supports $C_{w}$ at $w$ also supports $S$ at the same point, i.e., the linear functional $g(w) f+f(w) g$ attains its norm at $w$, and it follows from $w=\|u+v\|^{-1}(u+v)$ that we are in the hypothesis of Proposition 1.

(Another way to see that $H_{w}$ is the only closed hyperplane that supports $C_{w}$ at $w$ is to consider that $t=f(w) g(w)+g(w) f(x-w)+f(w) g(x-w)$ is the tangent hyperplane, in $E \times \mathbb{R}$, to $t=f(x) g(x)$ at the point $(w, f(w) g(w))$, and to consider that there is a translation of $C_{w}$ in the section of $t=f(x) g(x)$ by the hyperplane $t=f(w) g(w)$.)

Proposition 3. A real normed space $E$ of dimension $\geq 3$ is an i.p.s. if and only if, for any $u, v \in S$ and $f \in J u, g \in J v$,

$$
\exists r>0, \exists t>0:(f+r g)(u+t v)=\|f+r g\|\|u+t v\| .
$$

Proof. If $E$ is an i.p.s., it suffices to take $r=t=1$.

To prove the converse we can suppose that $E=\mathbb{R}^{3}$. Let $L$ be a 2-dimensional subspace of $E$ and $u, v \in S \cap L, f \in J u, g \in J v$, be such that $u \neq \pm v$ and $f \neq \pm g$. Then, $m=\operatorname{ker} f \cap \operatorname{ker} g$ is a homogeneous straightline and $S$ is supported at $u, v$ and another point $\|u+t v\|^{-1}(u+t v)$ between them, by straight lines parallel to $m$. Moreover, applying the hypothesis to $f \in J u$, and $-g \in J(-v)$, we obtain that $S$ is also supported by a straight line parallel to $m$ at some point between $u$ and $-v$.

From the above, it follows easily that parallel straight lines to $m$ support $S$ at every point of $S \cap L$. Since $L$ is an arbitrary 2-dimensional subspace, this is a wellknown characteristic property of ellipsoids, due to Brunn, Blaschke and Kakutani (see, e.g., [1, p. 99]).

Proposition 4. A real normed space $E$ of dimension $\geq 3$ is an i.p.s. if and only if, for any $u, v \in S$ and $f \in J u, g \in J v$, there exists $t>0$ such that the real function $x \in S \rightarrow f(x) g(x) \in \mathbb{R}$ attains its maximum at the point $\|u+t v\|^{-1}(u+t v)$.

Proof. If $E$ is an i.p.s., take $t=1$. Conversely, we have seen in the proof of Proposition 2 that the hypothesis implies that the linear functional $g(u+t v) f+$ $f(u+t v) g$ attains its norm at $\|u+t v\|^{-1}(u+t v)$. We are, therefore, in the hypothesis of Proposition 3 for $r=f(u+t v)(g(u+t v))^{-1}$.

\section{ACKNOWLEDGEMENT}

We thank the referee of this paper for his/her kind and accurate suggestions, especially those relative to Proposition 2.

\section{REFERENCES}

[1] D. AmiR, "Characterizations of inner product spaces", Birkhauser Verlag, Basel, 1986. MR 88m: 46001

[2] M. Baronti, Su alcuni parametri degli espazi normati, Bol. Unione Mat. Ital., B(5) 18 (1981), 1065-1085. MR 83b:46017 
[3] M.M. DAY, Some characterizations of inner product spaces, Trans. Amer. Math. Soc., 62 (1947), 320-337. MR 9:192c

[4] R.C. James, Inner products in normed linear spaces, Bull. Amer. Math. Soc., 53 (1947), 559-566. MR 9:42d

Departamento de Matemáticas, Universidad de Extremadura, 06071 Badajoz, Spain

E-mail address, C. Benítez: cabero@unex.es 